\title{
A technique to package Fiber Bragg Grating Sensors for Strain and Temperature Measurements
}

\author{
Uilian Dreyer, Kleiton de Morais Sousa, Jonas Somenzi, Ivo de Lourenço Junior, \\ Jean Carlos Cardozo da Silva \\ Electrical Engineering Graduated Program - PPGEE, Federal University of Technology - Paraná, Pato \\ Branco, Brazil, ZIP 85503-390. E-mail:\{ kleitonsousa, lourenco, jeanccs\}@utfpr.edu.br \\ Valmir de Oliveira, Hypolito José Kalinowski \\ Graduate Program on Electrical Engineering and Applied Computer Science - CPGEI, Federal University of \\ Technology - Paraná, Curitiba, Brazil, ZIP 80230-901.E-mail: \{valmir, hjkalin\}@utfpr.edu.br
}

\begin{abstract}
This paper reports an effective method of packaging a fiber Bragg grating (FBG) for the simultaneous measurement of temperature and strain. The technique consists of embedding two fiber Bragg grating sensors inside a polymeric material with different geometrical characteristics. The mechanical and thermal characterizations of the optical transducer were performed. Then a matrix equation used measurements of the wavelength shifts from the two sensors and yielded information about the temperature and strain coefficients. The preliminary results demonstrate the feasibility of the encapsulation technique allowing measurements of temperature and strain in smart structures and harsh environments. The experimental procedure provides robustness to the sensor and the matrix equation approach has the potential to determine simultaneously the strain and temperature coefficients.
\end{abstract}

Index Terms - Fiber Bragg gratings, packaging, strain and temperature measurement.

\section{INTRODUCTION}

The advancement of all the areas in science and technology depends on performance of the measuring processes that are necessary while investigating the corresponding natural phenomena. Therefore, in most applications, a sensor should have a reduced size and mass and be capable of measuring parameters without failure in a wide range of hostile environments and situations. In addition, it must be immune to electromagnetic interferences, have a high sensitivity and an acceptable resolution, as well as a low acquisition cost and maintenance.

It is difficult to find a device like those, but the optical fiber sensors have a large set of such characteristics, making them appropriate for a series of applications. The use of such sensors in the industrial, military, chemical, petrochemical, telecom, medical and aerospace sectors illustrates the diversity of measurands that can be detected with them, while their flexibility and adaptability to several fields of technology can be considered as additional characteristics [1]-[4].

One can mention other distinguished characteristics of those sensors, namely, low transmission 
losses (considering the distance between the point of measurement and the interrogation system, which can reach several kilometers); multiplexing capability; electrical passivity; measurand results coded into wavelength or intensity, and the possibility of using the same fiber as the sensor element and the communication channel.

Many projects involving the concept of smart structures would preferably use fiber optic sensors. In smart structures they can be found inside the beams of bridges, and also in applications to monitor the convergence of tunnels, the structure of dams, heritage structures and pipelines, among others [5]-[8].

Since the sensor can be appropriately inserted in the structures, the information at the local point of measurement could be directly obtained in a quasi-distributed configuration, avoiding the influence of external disturbances as it is the case when the sensor is installed on the surface of the structure or at a point where the measurand would be indirectly obtained.

For this application, fiber Bragg gratings (FBG) form a class of sensors that is being widely used. The description of its embedded use in smart structures can be found in the scientific literature [9][13], to name a few.

Fiber Bragg gratins are periodic structures imprinted usually in the core of an optical fiber that cause the refractive index to have a periodic modulation along its length. A beam of light guided in the core of the fiber will then be gradually diffracted by each of the periods of the modulation and the wavelengths that satisfy the Bragg condition will constructively add in the counter-propagating direction. This phenomenon will produce a signal reflected by the FBG that has an optical spectrum with a band centered at approximately the wavelength that satisfied the condition [14].

These structures can be represented by a set of transversal consecutive planes having the same disturbed refractive index in the core. From the characteristics of those planes, like their magnitude, period and envelope of the refractive index along the FBG, one is able to tune the coupling of the propagating mode to another counter propagating mode at a certain wavelength. In this case, the Bragg wavelength is represented by:

$$
\lambda_{\mathrm{B}}=2 \eta_{\mathrm{eff}} \Lambda
$$

where $\lambda_{B}$ is the Bragg wavelength, or resonance wavelength, $\eta_{\text {eff }}$ is the effective refractive index of the incident mode in the FBG and $\Lambda$ is the period of the modulation along the FBG.

Any effect that induces changes on the properties of the optical fiber with the FBG, like mechanical or thermal changes, may cause changes in the period or in the refractive index and this will shift the resonance wavelength. The changes in the FBG geometry and in the refractive index may force variations in the phase, intensity and polarization of the propagating signal. This dependence allows the FBG to be used as sensors that measure such disturbances.

In order to determine the sensitivity to those disturbances, equation (1) can produce the differential that represents the behavior of the Bragg wavelength with disturbances in temperature $(T)$ and length 
deformation of the sensor $(l)$. The change in the resonance wavelength is then given by equation (2) after developing the differential of equation (1):

$$
B=2\left(\frac{\partial_{\text {eff }}}{\partial I}+e_{\text {eff }} \frac{\partial}{\partial I}\right) I+2\left(\frac{\partial e_{\text {eff }}}{\partial T}+\text { eff } \frac{\partial}{\partial T}\right) T
$$

where $l$ is the length of the FBG and $T$ is the temperature. $\left(\partial n_{\text {eff }} / \partial l\right)$ represents the sensitivity of effective index to length changes of $\Delta L$, or the elasto-optic properties of the fiber, while $\left(\partial n_{\text {eff }} / \partial T\right)$ is related to the sensitivity of the index to thermal changes of $\Delta T$, or the thermo-optic properties. ( $/ T$ ) is related to the thermal expansion sensitivity of the sensor and $(/ L)$, to the length change in the FBG period caused by a length change.

In (2), a change in $\Delta \lambda_{B}$ is caused by mechanical and thermal disturbances in the sensor, either changing the refractive index or the period of the FBG. Therefore, the monitoring of the spectrum of the FBG reflected signal would allow the determination of this shift. Since the sensor is sensitive to both types of perturbations, one cannot distinguish if the wavelength shift was caused by a mechanical change or a thermal one. Kersey in [15] states that is unfortunate that, even having this immense breadth of potential application, the sensors do also tend to exhibit serious cross-sensitivity issues. This can be illustrated by the number of researchers that have written papers on the elimination of temperature dependence of a strain gauge, or a pressure sensor, or likewise, the effects of polarization on the sensitivity of such sensors. These practical limitations of fiber sensors - indeed, while interesting to be experimentally and theoretically explored, often result in fiber sensors providing weak competitive advantages over conventional sensor systems, thus limiting their value. This issue is, of course, key to the sustainable success of a fiber optic sensor in any true commercial application. A few techniques have been used to overcome such inconvenient cross-sensitivity [16]-[18].

In this work, an encapsulation process is described such as to be used with an FBG for the simultaneous measurement of temperature and strain. It is based on the work described Mokhtar et al. [10] and is theoretically based on the idea that a change in temperature and strain in the sensor causes a shift of the resonance wavelength through the effects of mechanical and thermal expansion, and the thermo-optic and elasto-optic effect on the refractive index.

\section{SIMULTANEOUS MEASUREMENT OF TEMPERATURE AND STRAIN}

One of the drawbacks using FBGs to measure temperature and strain is the cross-sensitivity that does not allow one to distinguish the measurands while determining the Bragg wavelength shift.

The change in Bragg wavelength can be written in a more compact form as a function of strain and temperature changes by:

$$
\Delta \lambda_{B}(\varepsilon, \mathrm{T})=K_{s} \Delta \varepsilon+K_{T} \Delta T
$$


Where $\Delta \lambda_{B}$ represents the change in Bragg wavelength, $K_{s}$ is the strain sensitivity and $K_{T}$ is the temperature sensitivity; the changes in strain and temperature are represented by $\Delta \varepsilon$ and $\Delta T$, respectively.

When a second FBG subject to the same strain and temperature, one can determine the Bragg wavelength change of each sensor from the matrix form of equation (3):

$$
\left[\begin{array}{l}
\Delta \lambda_{B 1} \\
\Delta \lambda_{B 2}
\end{array}\right]=\left[\begin{array}{ll}
K_{\varepsilon 1} & K_{T 1} \\
K_{\varepsilon 2} & K_{T 2}
\end{array}\right]\left[\begin{array}{c}
\Delta \varepsilon \\
\Delta T
\end{array}\right]
$$

The indexes 1 and 2 refer to the first and second FBG, respectively.

The response of both FBGs that have different sensitivities can be combined into a linear system and be solved to produce the temperature and strain change values. It is natural to think that the same system can be extended to any number of sensors.

In this case with two sensors, one can find $\Delta \varepsilon$ and $\Delta T$ by solving equation (4):

$$
\left[\begin{array}{c}
\Delta \varepsilon \\
\Delta T
\end{array}\right]=\frac{1}{D}\left[\begin{array}{cc}
K_{T 2} & -K_{T 1} \\
-K_{\varepsilon 2} & K_{\varepsilon 1}
\end{array}\right]\left[\begin{array}{l}
\Delta \lambda_{B 1} \\
\Delta \lambda_{B 2}
\end{array}\right]
$$

where $D=K_{\varepsilon 1} K_{T 2}-K_{\varepsilon 2} K_{T 1}$ is the determinant of the sensitivity matrix in (4).

\section{EXPERIMENTAL SETUP}

The developed packaging is made of a Polypropylene-Copolymer (PP) with non-uniform dimensions such as to produce distinct effective temperature and strain coefficients for each of the FBGs. By doing this, the coefficients $K_{T 1}$ and $K_{T 2}$ present different values, as in the case of the strain coefficients $K_{\varepsilon 1}$ and $K_{\varepsilon 2}$, which differ from each other. With this in mind, the values of temperature and strain can be obtained from equation (5).

For the fabrication of the sensors, a steel mold is used (SAE1020). The mold is designed to have a shape with different heights in the two cavities of the PP piece that will embed the sensors. When the sensor is completed, the part is pressed downwards with a system having small rods of steel which should push the sensor to the outside. In addition, the mold has round corners to facilitate the removal of the sensor, and a fissure such as to accommodate the optical fiber during the fabrication process.

In Figure 1, the designed mold is depicted for the fabrication of the packaging. The lower side of the packaging has a height of $2.50 \mathrm{~mm}$ and a length of $40.00 \mathrm{~mm}$, while the other side has a height of $5.20 \mathrm{~mm}$ and a length of $30.00 \mathrm{~mm}$. Both FBGs are located in a central region in each of the cavities with those specified heights, as depicted in the schematic diagram in Fig. 2, where those dimensions are indicated. 


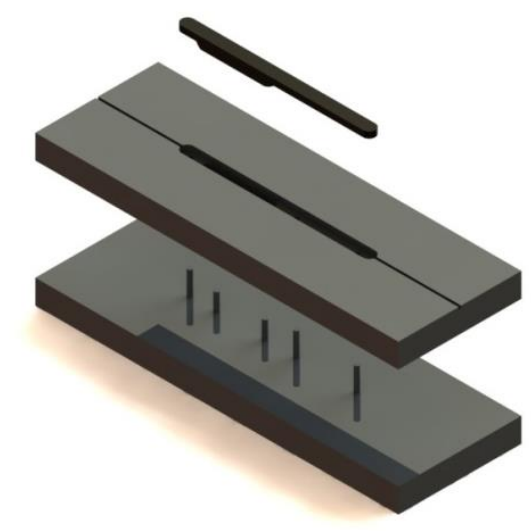

Figure 1 Designed mold for the fabrication of the sensors.

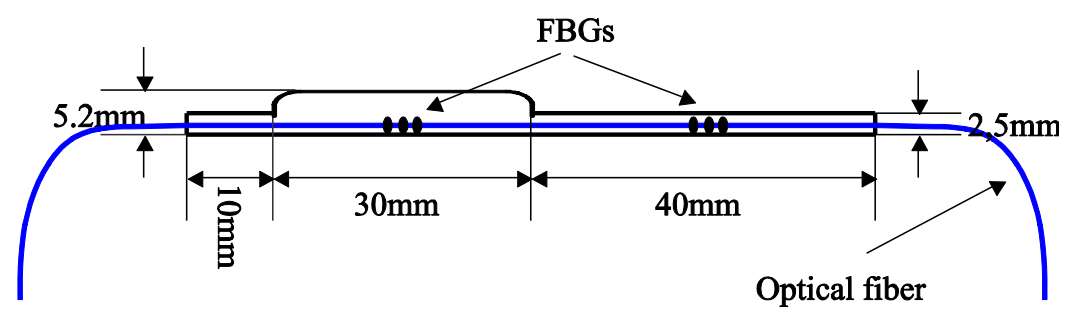

Figure 2 Schematic diagram of the sensor inside the designed mold where dimensions are indicated.

For the fabrication of the sensor, the PP spheres with a diameter of approximately $2.00 \mathrm{~mm}$ must be finely ground. This initial process guarantees that most of the spaces in the mold will be filled such as to avoid the occurrence of air bubbles inside it and a non-uniform surface on the packaging, since in this case it would modify the sensor response. The mold is preheated to a temperature of $200^{\circ} \mathrm{C}$ for a period of 50 minutes. The optical fiber is then positioned and the mold is filled with the ground PP. The set is treated in an oven at a temperature of $170^{\circ} \mathrm{C}$ for another 20 minutes until the melting of all the material. After additional 20 minutes, the mold is cooled with step reductions of $20^{\circ} \mathrm{C}$ in the temperature down to the temperature of the environment. This process is important to allow the accommodation of the PP material and to avoid the formation of bubbles near the surface of the sensor. The device is then removed from the mold with the help of the rods of steel. During the fabrication process, the spectrum of the reflected signal is monitored. The FBGs have initial Bragg wavelengths at $1542.00 \mathrm{~nm}$ and $1536.00 \mathrm{~nm}$ while at a temperature of $21^{\circ} \mathrm{C}$. It is shown that the resonance wavelengths shift to $1538.00 \mathrm{~nm}$ and $1528.00 \mathrm{~nm}$, respectively. This behavior is caused by the contraction of the sensor after the thermal treatment. The Bragg wavelength behavior also demonstrates that the FBG responds to changes while inside the packaging and also that there has been an adhesion of the fiber polymer coating to the polypropylene material. After removing the sensor from the mold, the surface is finished and the resulting device is depicted in Fig. 3. 


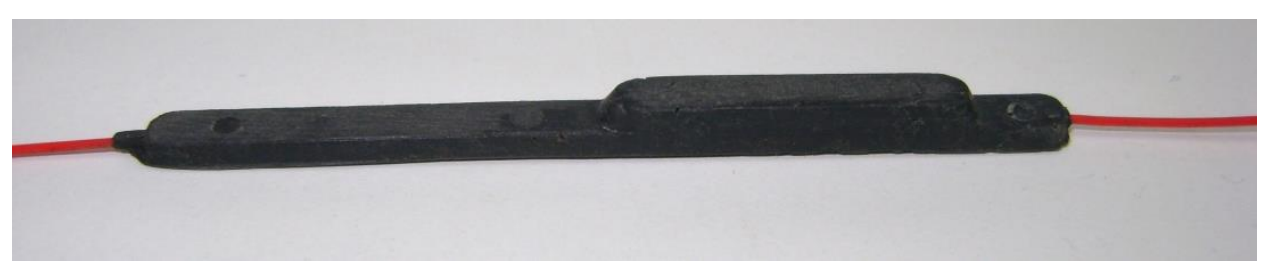

Figure 3 The fabricated sensor after the finishing process.

For the determination of the temperature and strain sensitivities, the resonance wavelengths were measured in experiments to separately evaluate the sensitivities. The sensor behavior was measured in a PolyScience ${ }^{\circledR}$ calibration bath with a resolution $0.1^{\circ} \mathrm{C}$ and the temperature varied from $20^{\circ} \mathrm{C}$ to $70^{\circ} \mathrm{C}$ in cycles repeated five times. The strain characterization was performed in a mechanical test machine with the sensor placed on a tensile strain test specimen. During the application of tension, the elongation of a strain-gage on the specimen was recorded against the applied force. This process was repeated five times. During the experiments, the FBGs were interrogated by a SI101 system with a wavelength resolution of $1 \mathrm{pm}$. The FBG interrogation system was produced by the HBM ${ }^{\circledR}$ company.

\section{RESULTS AND DISCUSSIONS}

The temperature calibration curves are shown in Fig. 4(a) and the strain curves are depicted in Fig. 4(b). The averaged points from the five calibrations are shown in the corresponding curves in those figures.

The experimental results present a linear response for the temperature and strain range, therefore the linear characteristic for the FBGs are maintained and is not altered by the packaging.

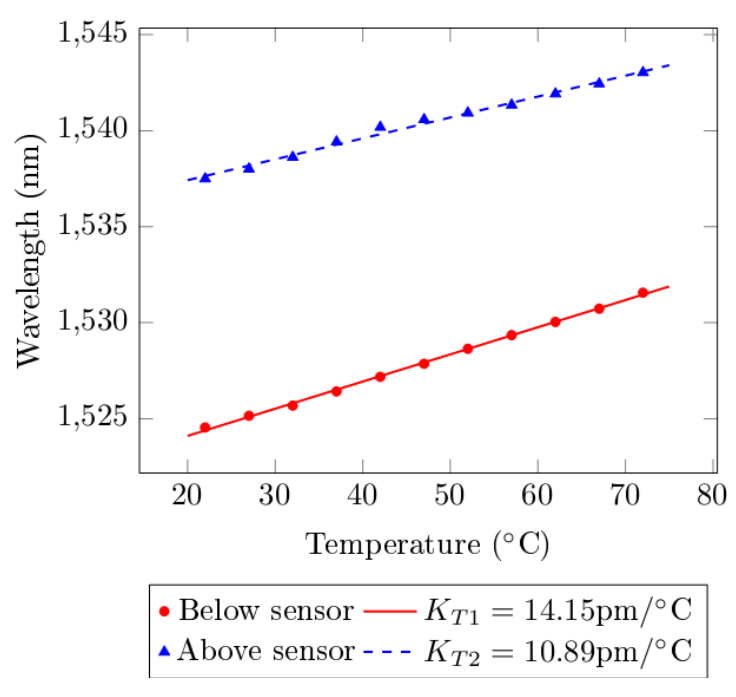

(a)

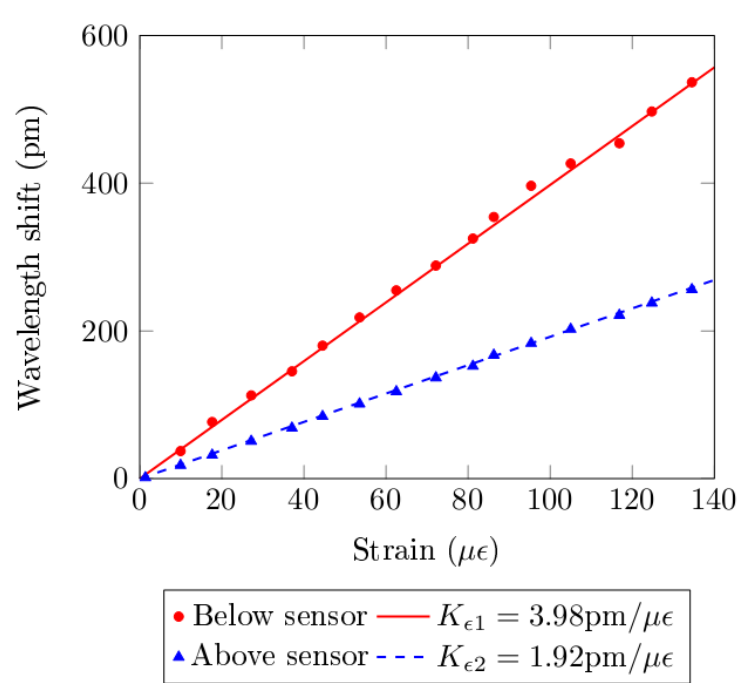

(b)

Figure 4 Results for the calibration of the sensor in terms of temperature (a) and strain (b). Experimental data (marks) are connected by best-fit lines whose sensitivities are given below each graph.

From the experimental results, linear best fits are performed and are also identified in Fig. 4(a) and 4(b). The linear fittings present a $R^{2}>0.99$ for the calibrations in temperature and strain. An 
important information concerning the linear fittings is that the sensitivities in the sensors located in the cavities with different dimensions have different sensitivities, namely, $K_{T 1}=14.15 \mathrm{pm} /{ }^{\circ} \mathrm{C}$ for the sensor in the lower part and $K_{T 2}=10.09 \mathrm{pm} /{ }^{\circ} \mathrm{C}$ for the sensor in the upper part. For the calibration of the sensor in the lower part, the sensitivity is calculated as $K_{\varepsilon 1}=3.98 \mathrm{pm} / \mu \varepsilon$, and presents a sensitivity of $K_{\varepsilon 2}=1.92 \mathrm{pm} / \mu \varepsilon$ for the sensor in the upper part. The obtained differences are due to the densities of the materials where the FBGs are placed. The lower part of the sensor has less material than the upper part.

The temperature and strain coefficients that have been experimentally obtained are used in equation (5) with the goal of producing converted values of temperature and strain corresponding to the wavelength measured values from the FBG sensors. The result is depicted Fig. 5, where the $x$-axis present the values in temperature units and the $y$ values in strain units. This plot allows one to demonstrate that it is possible to distinguish between temperature and strain results.

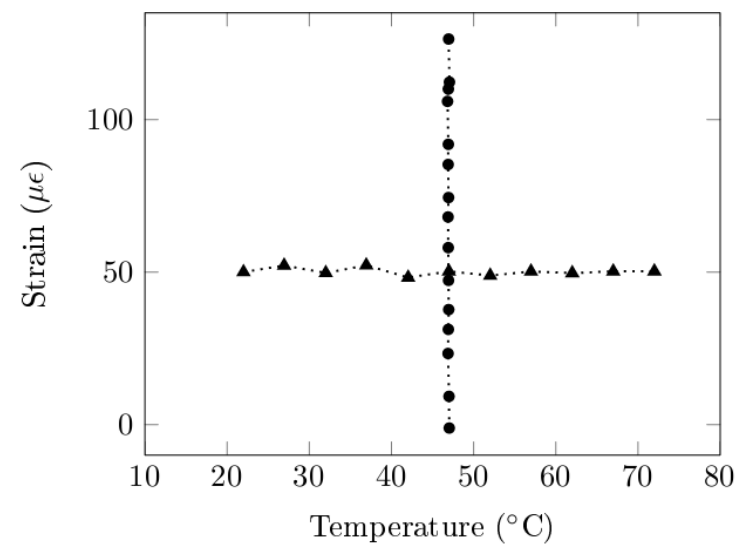

Figure 5 Sensor outputs as determined by equation (5) when subjected to various strain at a constant temperature or to varied temperature at a constant strain.

\section{CONCLUSION}

A packaging technique for FBG sensors has been reported, which was used to embed two FBG sensors in a polymer material. This encapsulation proved to be robust and capable of allowing the simultaneous measurement of strain and temperature through FBGs wavelength shifts measurements. The main contribution of this work resides in the development of a fabrication process of a packaging to embed the FBG sensors without the use of any type of adhesives. This represents an advantage under the point of view of precision characteristics of the sensor and it also allows the maintenance of the linear response of the FBG while measuring temperature or strain. In addition, to improve the accuracy of the measured values for strain and temperature is necessary to increase the difference between effective strain and temperature coefficients of the two sensors and this would be possible by using the proposed packaging design. 


\section{ACKNOWLEDGMENTS}

The authors acknowledge CAPES, CNPq, FINEP, SETI and Fundação Araucária for scholarships and funding. The FBG were fabricated in the Photo Refractive Devices Unit - UTFPR, an associate laboratory of INCT Fotonicom.

\section{REFERENCES}

[1] B. Culshaw, "Optical Fiber Sensor Technologies: Opportunities and-Perhaps-Pitfalls," Journal of Lightwave Technology, vol. 22, no. 1, pp. 39-50, Jan. 2004.

[2] K. T. V. Grattan and T. Sun, "Fiber optic sensor technology: an overview," Sensors and Actuators A: Physical, vol. 82, no. 1-3, pp. 40-61, May 2000.

[3] C. K. Kirkendall and A. Dandridge, "Overview of high performance fibre-optic sensing," Journal of Physics D: Applied Physics, vol. 37, no. 18, pp. R197-R216, 2004.

[4] B. Lee, "Review of the present status of optical fiber sensors," Optical Fiber Technology, vol. 9, no. 2, pp. 57-79, Apr. 2003.

[5] H. N. Li, D. S. Li, and G. B. Song, "Recent applications of fiber optic sensors to health monitoring in civil engineering," Engineering Structures, vol. 26, no. 11, pp. 1647-1657, 2004.

[6] B. Wang, J. G. Teng, L. D. Lorenzis, L.-M. Zhou, J. Ou, W. Jin, and K. T. Lau, "Strain monitoring of RC members strengthened with smart NSM FRP bars," Construction and Building Materials, vol. 23, no. 4, pp. 1698-1711, 2009.

[7] L. Rippert, J. Papy, M. Wevers, S. V. Huffel, and K. U. Leuven, "Fibre optic sensor for continuous health monitoring in CFRP composite materials," Smart Structures and Materials, vol. 4693, pp. 312-323, 2002.

[8] E. Mehrani, A. Ayoub, and A. Ayoub, "Evaluation of fiber optic sensors for remote health monitoring of bridge structures," Materials and Structures, vol. 42, no. 2, pp. 183-199, Apr. 2008.

[9] M. Majumder, T. K. Gangopadhyay, A. K. Chakraborty, K. Dasgupta, and D. K. Bhattacharya, "Fibre Bragg gratings in structural health monitoring-Present status and applications," Sensors and Actuators A: Physical, vol. 147, no. 1, pp. 150-164, 2008.

[10] M. R. Mokhtar, T. Sun, and K. T. V. Grattan, "Bragg Grating Packages With Nonuniform Dimensions for Strain and Temperature Sensing," IEEE Sensors Journal, vol. 12, no. 1, pp. 139-144, Jan. 2012.

[11] A. Kerrouche, W. J. O. Boyle, K. T. V. Grattan, J. W. Schmidt, and B. Taljsten, "Strain Measurement Using Embedded Fiber Bragg Grating Sensors Inside an Anchored Carbon Fiber Polymer Reinforcement Prestressing Rod for Structural Monitoring," IEEE Sensors Journal, vol. 9, no. 11, pp. 1456-1461, Nov. 2009.

[12] H. Ling, K. Lau, L. Cheng, and K. Chow, "Embedded fibre Bragg grating sensors for nonuniform strain sensing in composite structures," Measurement Science and Technology, vol. 16, pp. 2415-2424, 2005. 
[13] Y. J. Rao, "Recent progress in applications of in-fibre Bragg grating sensors," Opt. Lasers Eng., vol. 31, pp. 297-324, 1999.

[14] A. Othonos, "Fiber Bragg gratings," Review of Scientific Instruments, vol. 68, no. 12, p. 4309, 1997.

[15] A. D. Kersey, "Fiber optic sensors shine bright: industrial applications where FOS bring differentiated performance/value," vol. 8421, pp. 1-4, 2012.

[16] O. Frazão and J. L. Santos, "Simultaneous measurement of strain and temperature using a Bragg grating structure written in germanosilicate fibres," Journal of Optics A: Pure and Applied Optics, vol. 6, no. 6, pp. 553-556, Jun. 2004.

[17] L. Kuo and Z. Zhen'an, "A high sensitive fiber Bragg grating strain sensor with automatic temperature compensation," Chinese Optics Letters, vol. 7, no. 3, pp. 191-193, 2009.

[18] X. Shu, Y. Liu, D. Zhao, B. Gwandu, F. Floreani, L. Zhang, and I. Bennion, "Dependence of temperature and strain coefficients on fiber grating type and its application to simultaneous temperature and strain measurement.," Optics letters, vol. 27, no. 9, pp. 701-3, May 2002. 